\title{
INTENSITY CORRECTION IN TEXTURE MEASUREMENT OF POLYCRYSTALLINE THIN FILMS BY X-RAY DIFFRACTION
}

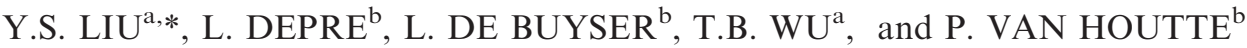 \\ ${ }^{a}$ School of Materials Science and Engineering, Jiao Tong University, \\ Shanghai 200030, China; ${ }^{b}$ Department of Metallurgy and Materials Engineering, \\ Katholieke Universiteit Leuven, B-3001 Leuven, Belgium
}

(Received 28 May 2003; In final form 5 June 2003)

\begin{abstract}
The tilting of a specimen may not only result in defocusing effect, but also in a change of irradiated volume during texture measurement for thin films and coatings by using X-ray diffraction. The influence on diffraction intensity has to be considered in order to obtain an accurate result of texture analysis. The effect of irradiated volume can be formulated as a function of the thickness and the linear absorption of a studied film material, the tilt angle of the specimen and the Bragg angle of diffraction, and then eliminated. In view of that the preparation of a randomly oriented specimen would be difficult, a simple and convenient method is hereby proposed to correct the defocusing effect from known data of existing bulk powder specimens with random orientation by interpolation (or extrapolation). The two correction methods were experimentally verified to be effective and reliable.
\end{abstract}

Keywords: Thin films; Texture measurement; X-ray diffraction; Irradiated volume; Defocusing effect; Intensity correction

\section{INTRODUCTION}

Thin film coatings have been widely used for a long time to improve surface properties of materials, for example tribological performance (Huang et al., 1994) and corrosion resistance (Dong et al., 1997). In the last decade many thin film materials such as thin film shape memory alloys (SMAs) (Krulevitch et al., 1996; Benard et al., 1998), bimetallic thin films, piezoelectric thin films (Van Lintel et al., 1988), ferroelectric and antiferroelectric films have enabled the development of innovative products such as sensors, actuators, and transducers in microelectronic-mechanical systems (MEMS) (Fujita, 1997). A number of these films are polycrystalline aggregates, the properties of which may be influenced by preferred orientations of crystallites (or textures) due to anisotropy of physical and mechanical properties of crystals. Hence a great attention is being paid to the investigation of textures in the thin films (Knight and Page, 1990; Kim and Oliveria, 1994; Petrenco, 1995; Park and Szpunar, 1998).

*Corresponding author. E-mail: ysliu@sh163.net 
The textures are most popularly measured by X-ray diffraction (XRD). However, the thin films in practical application usually have a smaller thickness than the X-ray penetration depth. This results in the variation of irradiated volume of specimens during the texture measurement. In order to accurately measure and analyze textures in thin films, Szpunar et al. (1993) provided a solution: the diffraction of film layer by using low incident angle, or the theoretical correction of X-ray penetration length (or irradiated volume) for conventional diffraction was utilized by Chateigner et al. (1992). Because the former method needs an additional measuring and analyzing program, we prefer to use here the latter principle but a different correction procedure. A further motivation for the present work is the fact that some film materials are multicomponent compounds or even have complex compositions. It may be difficult to obtain powder substances for preparing randomly oriented specimen, needless to say further considering about its metallurgical condition and process. For this reason a simple and convenient interpolation (or extrapolation) method was proposed to obtain the random intensities from the experimental data of some existing bulk powder specimens.

\section{CORRECTION FOR TEXTURE MEASUREMENT AND ANALYSIS IN THIN FILMS}

\subsection{Correction of Irradiated Volume}

Let us consider the diffraction of X-rays from a certain lattice plane in a film in such a case as symmetric optical geometry. If the film thickness is far smaller than the penetration depth of X-rays and the preferred orientation of crystallites (or texture) is not considered, the ratio of X-ray diffraction intensities, corresponding to the sample tilting angles: $\alpha \neq 0^{\circ}$ and $\alpha=0^{\circ}$, can be written as

$$
\frac{I_{\alpha \neq 0}}{I_{\alpha=0}}=\frac{1-\exp \left(-2 \mu^{*} t / \sin \theta \cos \alpha\right)}{1-\exp \left(-2 \mu^{*} t / \sin \theta\right)}
$$

where $\mu^{*}$ is the linear absorption coefficient of the film material, $t$ is the film thickness and $\theta$ is the Bragg angle of the diffraction plane. Equation (1) illuminates the variation in the diffracted intensity at $\alpha \neq 0^{\circ}$ with respect to the intensity at $\alpha=0^{\circ}$, caused by the change of effective penetrated thickness (or irradiated volume) of X-rays. The ratio value, $C_{v}=I_{\alpha \neq 0^{\circ}} / I_{\alpha=0^{\circ}}$, may be used for the correction of the irradiated volume, thereby called as the correction factor for irradiated volume. Dividing the intensity diffracted from a tilted specimen $\left(\alpha \neq 0^{\circ}\right)$ by the correction factor $C_{v}$ allows it normalized to the intensity in the case of $\alpha=0^{\circ}$ (or having the same irradiated volume as no tilt). It means that the effect of irradiated volume has already been deducted from the normalized intensity.

Taking the (110)-diffraction of a NiTi shape memory film $\left(\mu_{\rho}=115.4505 \mathrm{~cm}^{2} / \mathrm{g}\right)$ with $4.91 \mu \mathrm{m}$ thickness as an example (with $\mathrm{Cu}-\mathrm{K} \alpha$ radiation and $2 \theta=42.456^{\circ}$ ), and normalizing its diffraction intensity at $\alpha=0^{\circ}$ to 1 , the influence of sample tilting on intensity is shown in Fig. 1. One can see that the intensity becomes enlarged because the irradiated volume increases with sample tilting. 


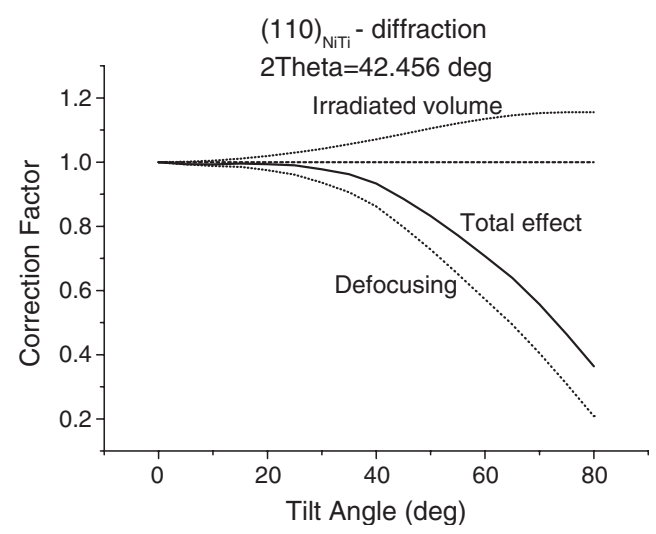

FIGURE 1 Effect of irradiated volume and defocusing on the intensity of (110)-diffraction in a NiTi film with $\mathrm{Cu}-\mathrm{K} \alpha$ radiation.

\subsection{Defocusing Correction}

The defocusing effect is caused by the fact that the irradiated surface layer deviates from the focusing sphere surface when a specimen is tilted, especially at high tilting angles. The theoretical analysis of the problem is very complex and maybe impossible. The most common method for determining the defocusing effect is to use a randomly oriented bulk powder specimen (so called as random standard specimen). Figure 2 shows some experimentally determined defocusing curves, which display the decrease of intensities diffracted from different lattice planes of two conventional bulk powder specimens of pure $\mathrm{Al}$ and $\mathrm{Cu}$ with the increase of tilting angle $\alpha$. However, in some cases, it is difficult to obtain such a random standard specimen as consistent with the measured specimen in microstructure, even though their chemical components are close to each other. Such as the example of SMAs, the phase structures for both specimens would be different at the measuring temperature due to phase transformation. Maybe, one was austenite and another was martensite. Hereby we corrected the defocalization by using the following interpolation (or extrapolation) method from the known data of existing bulk powder specimens. The defocusing effect depends only on tilting angle $\alpha$ of a specimen and the Bragg angle $\theta$ of diffraction, when the radiation target and the optical geometry of a given equipment are kept constant and the difference of surface morphology and internal constitutions among different specimens may be neglected. Thus, by keeping tilting angle $\alpha$ constant, one could find the variation of the intensities with the Bragg angle $\theta$ from the acquired data by means of polynomial fit. However, the type of material and the reflectivity of the selected lattice plane may influence the diffracted absolute intensities. In order to eliminate these effects, in the present work, the measured intensities were normalized as

$$
I_{\alpha=0^{\circ}}=1
$$

It was found that a good linear relation for the diffracted intensity versus Bragg angle $\theta$ appears at larger tilting, and the larger the tilting angle is, the smaller the spread around the linear relation, although more data are probably necessary to prove this by statistical means. Figure 3 presents an example, where the intensity variations 

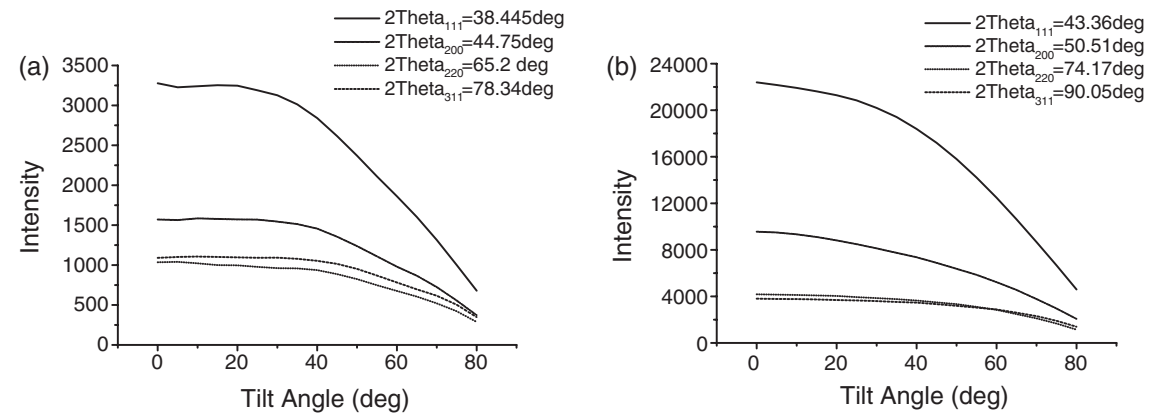

FIGURE 2 Reflected intensities of randomly oriented samples with $\mathrm{Cu}-\mathrm{K} \alpha$ radiation: (a) $\mathrm{Al}$ powder sample; (b) $\mathrm{Cu}$ powder sample.
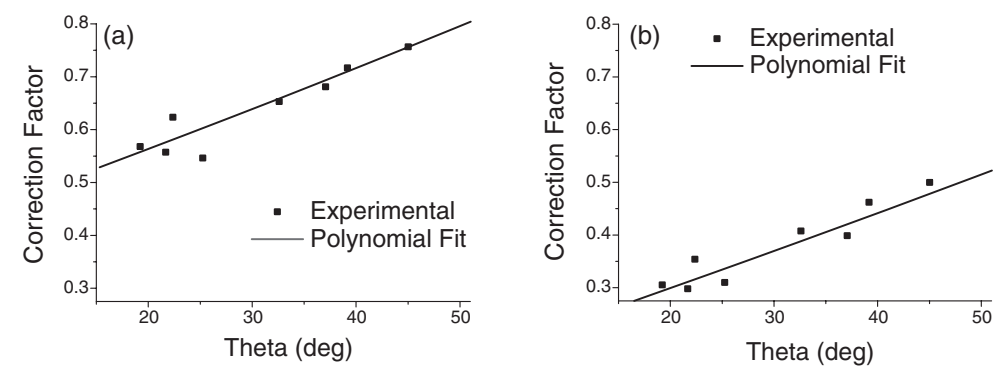

FIGURE 3 Polynomial fit of relationship between defocusing effect and Bragg angle $\theta$ with respect to a fixed tilting angle: $\alpha$ (a) $\alpha=60^{\circ}$; (b) $\alpha=75^{\circ}$.
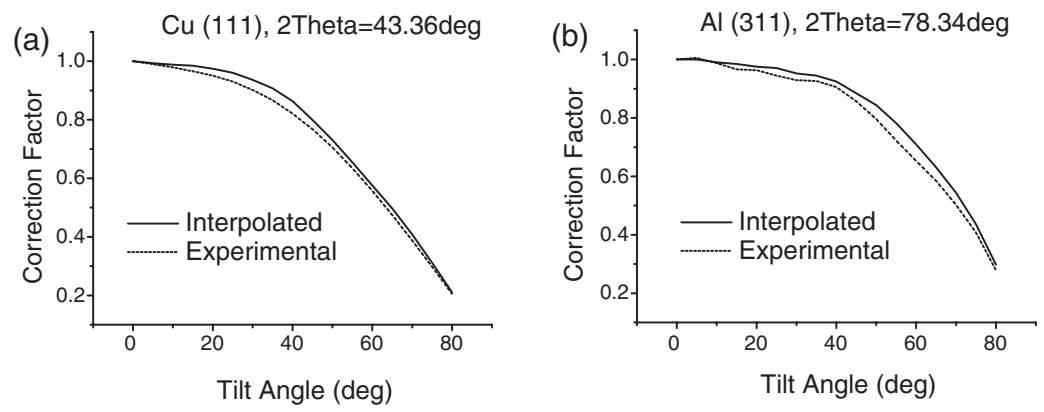

FIGURE 4 Experimental and interpolated defocusing effect: (a) $\mathrm{Cu}(111)$; (b) $\mathrm{Al}(311)$.

with the Bragg angle $\theta$ are quite linear at $\alpha=60$ and $75^{\circ}$ under the optically geometrical condition of the utilized diffractometer. From these curves of normalized intensity vs. $\theta$, one can then obtain the defocusing curve for any $\theta$ (or any diffraction), similar to the ones for $\mathrm{Al}$ and $\mathrm{Cu}$ diffractions as given in Fig. 2, by an interpolation (or extrapolation) method from enough experimental data.

Using the deduced function relation between diffraction intensity and Bragg angle, some recalculated correction factors of defocalization for $\mathrm{Al}$ and $\mathrm{Cu}$ diffractions are given in Fig. 4. One can see that these are in nice agreement with the experimental results. Fig. 1 also displays the interpolated effect of defocusing on (110) ${ }_{\mathrm{NiTi}}$-diffraction of the NiTi film specimen with $\mathrm{Cu}-\mathrm{K} \alpha$ radiation. As shown, the diffraction intensity 
decreases with the increase of tilting angle $\alpha$, caused by defocusing. The variation of normalized intensity vs. $\alpha$ can be used as the correction factor of defocusing for a fixed $\theta$, such as done in the above-mentioned correction of irradiated volume. Divided by the correction factor, a diffracted intensity, corresponding to $\alpha \neq 0^{\circ}$, will be normalized to the one at $\alpha=0^{\circ}$.

Combining both correction factors for defocusing and irradiated volume, the total correction factor is also illustrated in Fig. 1.

\section{EXPERIMENTAL VERIFICATION}

The incomplete pole figures (110), (200) and (211) with $\alpha \leq 80^{\circ}$ for the NiTi-film, as mentioned in the above chapter, were measured by using Schulz reflection method and $\mathrm{Cu}-\mathrm{K} \alpha$ radiation. In Fig. 5(a) the results are given directly from raw data. After correction by use of the total correction factors, including the defocusing and the irradiated volume correction, the corresponding pole figures are shown in Fig. 6(a). It can be determined from the two sets of pole figures that there are stronger (110)- and (111)-fibre textures in the normal direction (ND) to the surface in the film. As known, the error of experimentally determined pole figures will influence the precision of ODF (Orientation Distribution Function) analysis (Bunge, 1982).
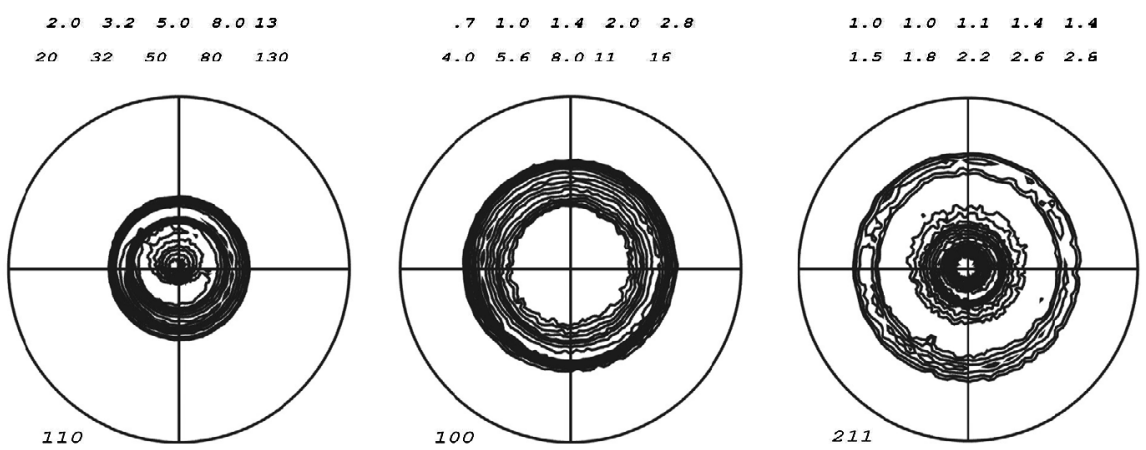

(a)
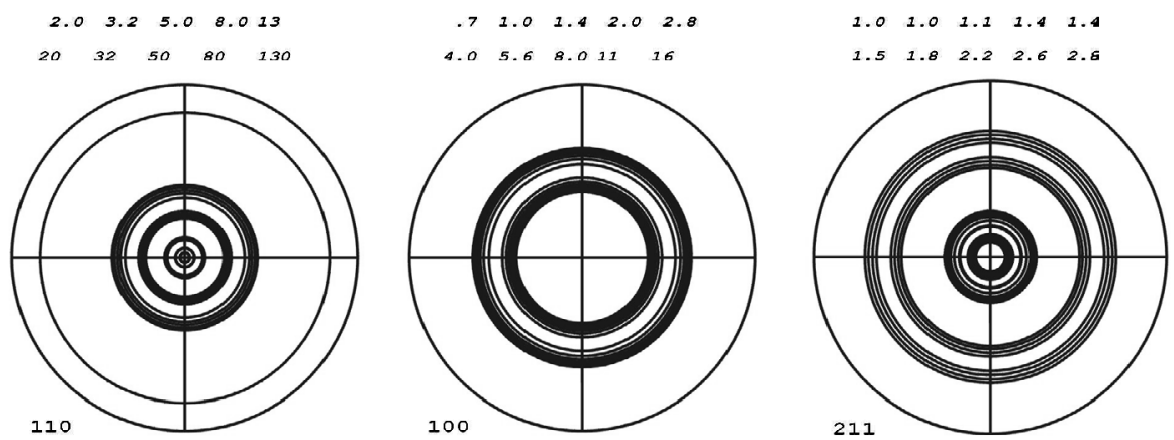

(b)

FIGURE 5 Pole figures without defocusing and irradiated corrections: (a) experimental; (b) recalculated. 

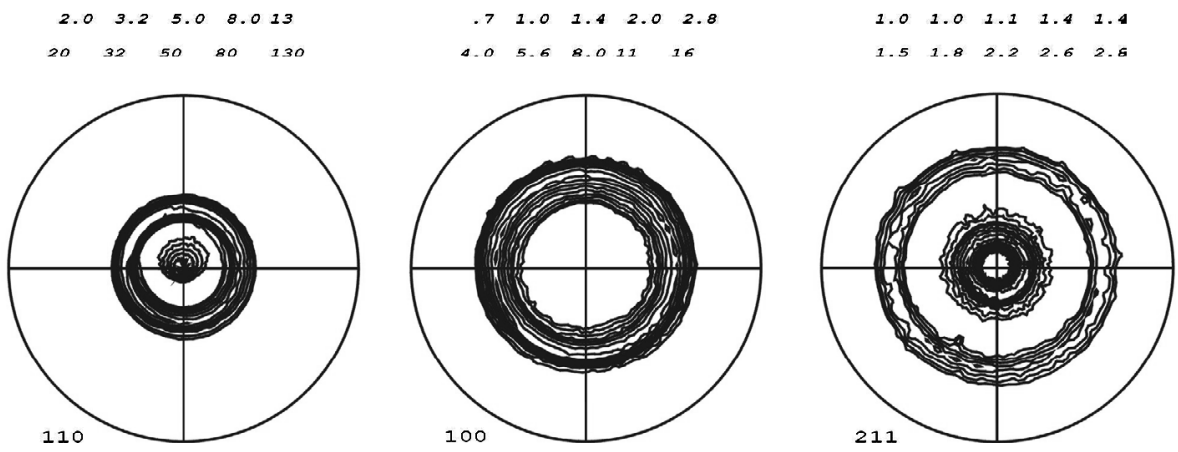

(a)
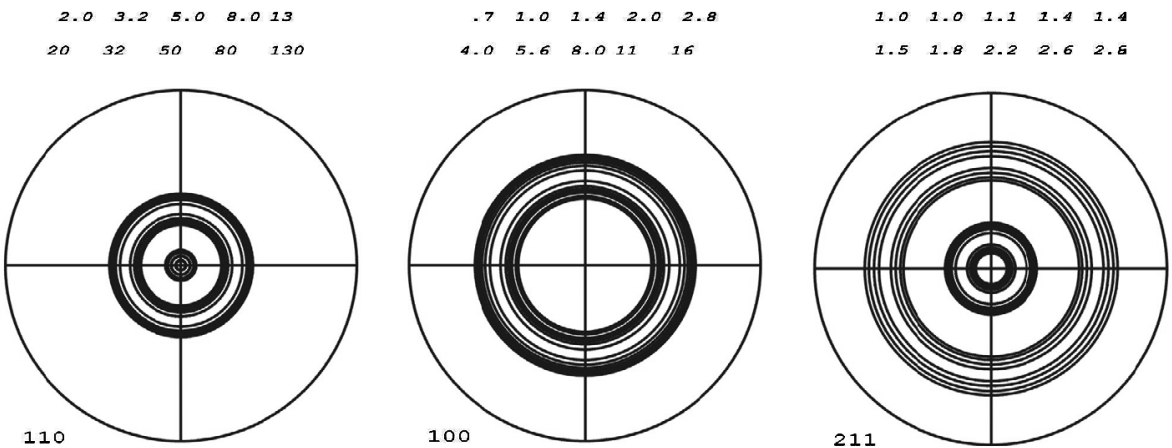

(b)

FIGURE 6 Pole figures after defocusing and irradiated corrections: (a) experimental; (b) recalculated.

The larger the error of original pole figures, the larger the distortion of recalculated results by ODF analysis. In order to compare the analyzed results before and after the above-mentioned correction, the recalculated pole figures $\left(\alpha \leq 80^{\circ}\right)$ for the two cases are given respectively in Figs. 5(b) and 6(b). For comparison, the experimental pole figures in Figs. 5(a) and 6(a) were normalized as

$$
\int_{0}^{2 \pi} \int_{0}^{\alpha_{\max }} p(\alpha \beta)_{\exp } \sin \alpha d \alpha d \beta=\int_{0}^{2 \pi} \int_{0}^{\alpha_{\max }} p(\alpha \beta)_{\text {recal }} \sin \alpha d \alpha d \beta
$$

where $p(\alpha \beta)_{\exp }$ is the experimental pole figure (raw or corrected) and $p(\alpha \beta)_{\text {recal }}$ is the recalculated one. From Figs. 5 and 6 we can see, although the distributions of the maximum in both results are similar, an additional strong ring appears near to $\alpha=80^{\circ}$ in the recalculated pole figure (110) only in Fig. 5(b), which is absent in the experimental one. The error between the experimental and recalculated pole figures can be quantitatively estimated by

$$
\text { Error }=\frac{\int_{0}^{2 \pi} \int_{0}^{\alpha_{\max }}\left|p(\alpha \beta)_{\exp }-p(\alpha \beta)_{\text {recal }}\right|^{2} \sin \alpha d \alpha d \beta}{\int_{0}^{2 \pi} \int_{0}^{\alpha_{\max }}\left|p(\alpha \beta)_{\exp }\right|^{2} \sin \alpha d \alpha d \beta}
$$


TABLE I The error between the experimental and recalculated pole figures

\begin{tabular}{lrrr}
\hline & $(110)$ & $(200)$ & $(211)$ \\
\hline Uncorrected (Fig. 5) & $16.4 \%$ & $5.1 \%$ & $21.9 \%$ \\
Corrected (Fig. 6) & $7.0 \%$ & $3.6 \%$ & $10.9 \%$ \\
\hline
\end{tabular}

The calculated errors for Figs. 5 and 6 are listed in Table I, which indicates an improved result after correction.

\section{CONCLUSIONS}

1. A lot of the technologically used thin films and coatings have a thickness smaller than the penetration ability of X-rays. In order to measure and analyze the textures in the materials by XRD, one has to consider the influence of not only defocusing but also irradiated volume on diffracted intensities caused by specimen tilting.

2. When the absorption of X-rays in the thin films and coatings is known, the effect of irradiated volume on diffracted intensities can be theoretically predicted and eliminated.

3. If a randomly oriented standard specimen is difficult to prepare, the correction factor for defocusing effect can be approximately determined by the interpolation (or extrapolation) method proposed in the present work.

\section{Acknowledgments}

Financial support for this work has been obtained from the Flemish Government, Belgium through the contract BIL/99/02 and from the fund for technological development of Shanghai Science and Technology Committee, China (project code: 995207010). The authors would like to express them the most heartfelt acknowledgment.

\section{References}

Benard, W.L., Kahn, H. and Heuer, H. (1998). Thin-film shape-memory alloy actuated micropump. J. MEMS., 7(2), 245-251.

Bunge, H.J. (1982). Texture Analysis in Materials Science. Butterworths, London.

Chateigner, D., Germi, P. and Pernet, M. (1992). Texture analysis by the Schulz reflection method: defocalization correction for thin films. J. Appl. Cryst., 25, 766-769.

Dong, H., Sun, Y. and Bell, T. (1997). Enhanced corrosion resistance of duplex coating. Surf. Coat. Tech., 90(1-2), 90-101.

Fujita, H. (1997). A decade of MEMs and its future. In: Proc. of IEEE Micro-Electro-Mechanical Systems, pp. $1-8$.

Huang, Z.P., Sun, Y. and Bell, T. (1994). Friction behavior of TiN, CrN and (TiAl)N coatings. Wear, 173(1-2), 13-20.

Kim, Y.K. and Oliveria, M. (1994). Magnetic-properties and texture of sputtered $\mathrm{Fe} / \mathrm{Fe}_{3} \mathrm{O}_{4}$ multilayer films. IEEE Transactions on Magnetics, 30(3), 1316-1323.

Knight, J.C. and Page, T.F. (1990). The fine-scale microstructure of thin hard TiN and TiC coatings on steels. Thin Solid Films, 193(1-2), 431-441.

Krulevitch, P.A., Lee, P., Ramsey, P.B., Trevino, J.C., Hamilton, J. and Northrup, M.M. (1996). Thin film shape memory alloy microactuators. J. MEMS, 5(4), 270-281. 
Park, H. and Szpunar, J.A. (1998). The role of texture and morphology in optimizing the corrosion resistance of zinc-based electrogalvanized coatings. Corrosion Science, 40(4-5), 525-545.

Petrenco, P.A. (1995). Relation between structural and electronic-properties of $\mathrm{SnO}_{2}$ polycrystalline thin-films prepared by the aerosol MOCVD technique. Thin Solid Films, 263(1), 122-126.

Szpunar, J.A., Ahlroos, S. and Tavernier, Ph. (1993). Method of measurement and analysis of texture in thin films. Journal of Materials Science, 28(9), 2366-2376.

Van Lintel, H.T.G., Van de Pol, F.C.M. and Bouwstra, S. (1988). A piezoelectric micropump based on micromaching of silicon. Sensors and Actuators, 15, 153-167. 